\title{
Attractor Solutions in Lorentz Violating Scalar-Vector-Tensor Theory
}

\author{
Arianto $^{(1,2,3)}$ * Freddy P. Zen ${ }^{(1,2)}$ 丹 Triyanta ${ }^{(1,2)}$ 团 and Bobby E. Gunara ${ }^{(1,2)}$ \\ (1) Theoretical Physics Lab., THEPI Devision, \\ and \\ ${ }^{(2)}$ Indonesia Center for Theoretical and Mathematical Physics (ICTMP) \\ Faculty of Mathematics and Natural Sciences, \\ Institut Teknologi Bandung, \\ Jl. Ganesha 10 Bandung 40132, INDONESIA. \\ (3) Department of Physics, Udayana University \\ Jl. Kampus Bukit Jimbaran Kuta-Bali 80361, INDONESIA.
}

\begin{abstract}
We investigate properties of attractors for scalar field in the Lorentz violating scalar-vector-tensor theory of gravity. In this framework, both the effective coupling and potential functions determine the stabilities of the fixed points. In the model, we consider the constants of slope of the effective coupling and potential functions which lead to the quadratic effective coupling vector with the (inverse) power-law potential. For the case of purely scalar field, there are only two stable attractor solutions in the inflationary scenario. In the presence of a barotropic fluid, the fluid dominated solution is absent. We find two scaling solutions: the kinetic scaling solution and the scalar field scaling solutions. We show the stable attractors in regions of $(\gamma, \xi)$ parameter space and in phase plane plot for different qualitative evolutions. From the standard nucleosynthesis, we derive the constraints for the value of the coupling parameter.

PACS numbers: $98.80 . \mathrm{Cq}$
\end{abstract}

\section{INTRODUCTION}

One of the interesting and widely exploited cosmological models is the scalar-tensor theory of gravity, the theory of a scalar field coupled to gravity. A remarkable phenomenon such as inflation, is usually discussed in the frame of this model. Recently, however, a scenario of implementing local Lorentz violation in a gravitational setting is considered to imagine the existence of a tensor field with a non-vanishing expectation value, and then to couple this tensor to gravity or matter fields. The vector field picks out a preferred frame at each point in spacetime, and any matter fields coupled to it will experience a violation of local Lorentz invariance [1, 2]. In a cosmological background, such a vector field acts to rescale the effective value of Newton's constant [3]. Moreover, from the study of the spontaneous breaking of Lorentz symmetry due to a vector field [4, 5, 6, 7], many current experiments and observations [8, 9, 10, 11, 12] can be explained. In Ref. [13] it has been studied the late time evolution of the vector field perturbation and its effects on cosmological observables. They found that the CMB and matter power spectra are modified.

More recently, a great interest has been devoted to study cosmological inflation in the framework of Lorentz violating scalar-vector-tensor theory of gravity [14]. They have shown that the Lorentz violating vector affects the

\footnotetext{
*Electronic address: feranie@upi.edu

${ }^{\dagger}$ Electronic address: fpzen@fi.itb.ac.id

${ }^{\ddagger}$ Electronic address: triyanta@fi.itb.ac.id

$\S$ Electronic address: bobby@fi.itb.ac.id
}

dynamics of the inflationary model. One of the interesting feature of this scenario, is the exact Lorentz violating inflationary solutions in the absence of the inflaton potential. In this case, the inflation is completely associated with the Lorentz violation. Depending on the value of the coupling parameter, the three kinds of exact solutions are found: the power law inflation, de Sitter inflation, and the superinflation. Moreover, the dynamics of superinflation in the context of Loop Quantum Cosmology, in which the Friedmann equation is modified by the presence of inverse volume corrections has been studied, recently [15].

The dynamical system of the scalar field with canonical Lagrangian has been widely studied [16, 17], among which the global structure of the phase plane has been investigated and various critical points and their physical significances have been identified and manifested. General properties of attractors for scalar-field dark energy scenarios which possess cosmological scaling solutions have been studied in Ref. [18] (see also [19]).

Cosmological attractor solutions have been found and studied by several authors for various classes of potentials. The main purpose of this paper is to show that it is possible to find attractor solutions in the Lorentz violating scalar-vector-tensor models in which both the effective coupling function and the potential function are specified, and their relation matters. In other words, we will find a class of models in which the dynamics of the system dependents on the effective coupling function and the potential function, as well as on their relation. More specific, we consider the model in which the slope of the effective coupling vector and the potential in the Lorentz violation are constants. Then we find the Lorentz vio- 
lating model with the quadratic effective coupling vector and the inverse or power-law-potential. The quintessence scenario with this potential has been well studied in the conventional universe [20, 21]. In the case of a tachyon field the corresponding potential is given by $V=V_{0} \phi^{-2}$ [22, 23, 24]. The dynamical attractor of the cosmological system has been employed to make the late time behaviors of the model insensitive to the initial condition of the field and thus alleviates the fine tuning problem. In quintessence models, the dynamical system has tracking attractor that makes the quintessence evolves by tracking the equation of state of the background cosmological fluid so as to alleviating the fine tuning problem [16, 17, 20].

This paper is organized as follows. In Section II we set down the general formalism for the scalar-vector-tensor theory where the Lorentz symmetry is spontaneously broken due to the unit-norm vector field. We derive the governing equations of motion for the canonical Lagrangian of the scalar field. In Section [II we study the attractor solutions for the purely scalar field. In Section IV] we extend our analysis in the presence of the barotropic fluid. The critical points of the system and their stability are presented. The final Section is devoted to the conclusions. In the Appendix, we present the stability of the fixed point in which both the slope of effective coupling function and potential function are constant parameters.

\section{LORENTZ VIOLATING SCALAR-VECTOR-TENSOR}

In the present section, we develop the general reconstruction scheme for the scalar-vector-tensor gravitational theory. We will consider the properties of general four-dimensional universe, i.e. the universe where the four-dimensional space-time is allowed to contain any non-gravitational degree of freedom in the framework of Lorentz violating scalar-tensor-vector theory of gravity. Let us assume that the Lorentz symmetry is spontaneously broken by getting the expectation values of a vector field $u^{\mu}$ as $<0\left|u^{\mu} u_{\mu}\right| 0>=-1$. The action can be written as the sum of three distinct parts:

$$
S=S_{g}+S_{u}+S_{\phi},
$$

where the actions for the tensor field $S_{g}$, the vector field $S_{u}$, and the scalar field $S_{\phi}$, respectively, are given by

$$
\begin{aligned}
S_{g}= & \int d^{4} x \sqrt{-g} \frac{1}{16 \pi G} R \\
S_{u}= & \int d^{4} x \sqrt{-g}\left[-\beta_{1} \nabla^{\mu} u^{\nu} \nabla_{\mu} u_{\nu}-\beta_{2} \nabla^{\mu} u^{\nu} \nabla_{\nu} u_{\mu}\right. \\
& -\beta_{3}\left(\nabla_{\mu} u^{\mu}\right)^{2}-\beta_{4} u^{\mu} u^{\nu} \nabla_{\mu} u^{\alpha} \nabla_{\nu} u_{\alpha} \\
& \left.+\lambda\left(u^{\mu} u_{\mu}+1\right)\right] \\
S_{\phi}= & \int d^{4} x \sqrt{-g} \mathcal{L}_{\phi}
\end{aligned}
$$

In the above $\beta_{i}(\phi)(i=1,2,3,4)$ are arbitrary parameters and $\mathcal{L}_{\phi}$ is the Lagrangian density for scalar field, expressed as a function of the metric $g_{\mu \nu}$ and the scalar field $\phi . \lambda$ is a Lagrange multiplier. Then, the action (1) describes the scalar-vector-tensor theory. For the timelike vector field, we impose a constraint

$$
u^{\mu} u_{\mu}=-1 \text {. }
$$

Here, we take $u^{\mu}$ as the dimensionless vector, and accordingly, $\beta_{i}$ has the dimension of mass squared. Thus, $\beta_{i}{ }^{1 / 2}$ gives the mass scale of symmetry breakdown. The preferred frame determined by the vector $u^{\mu}$ differs from the CMB rest frame and the alignment of these frames had been achieved during the cosmic expansion as is explained in the Appendix of Ref. 14]. In this setup, the preferred frame is selected through the constrained vector field $u^{\mu}$ and this leads to violating the Lorentz symmetry.

For the background solutions, we use the homogeneity and isotropy of the universe spacetime

$$
d s^{2}=-\mathcal{N}^{2}(t) d t^{2}+e^{2 \alpha(t)} \delta_{i j} d x^{i} d x^{j},
$$

where $\mathcal{N}$ is a lapse function. The scale of the universe is determined by $\alpha$. We take the constraint

$$
u^{\mu}=\left(\frac{1}{\mathcal{N}}, 0,0,0\right)
$$

where $\mathcal{N}=1$ is taken into account after the variation. Varying the action (1) with respect to $g^{\mu \nu}$, we have field equations

$$
R_{\mu \nu}-\frac{1}{2} g_{\mu \nu} R=8 \pi G T_{\mu \nu}
$$

where $T_{\mu \nu}=T_{\mu \nu}^{(u)}+T_{\mu \nu}^{(\phi)}$ is the total energy-momentum tensor, $T_{\mu \nu}^{(u)}$ and $T_{\mu \nu}^{(\phi)}$ are the energy-momentum tensors of vector and scalar fields, respectively, defined by the usual formulae

$$
T_{\mu \nu}^{(k)}=-2 \frac{\partial \mathcal{L}^{(k)}}{\partial g^{\mu \nu}}+g_{\mu \nu} \mathcal{L}^{(k)}, \quad k=u, \phi .
$$

The time and space components of the total energymomentum tensor are given by

$$
T_{0}^{0}=-\rho_{u}-\rho_{\phi}, \quad T_{i}^{i}=p_{u}+p_{\phi},
$$

where the energy density and pressure of the vector field are given by

$$
\begin{aligned}
& \rho_{u}=-3 \beta H^{2}, \\
& p_{u}=\left(3+2 \frac{H^{\prime}}{H}+2 \frac{\beta^{\prime}}{\beta}\right) \beta H^{2}, \\
& \beta \equiv \beta_{1}+3 \beta_{2}+\beta_{3} .
\end{aligned}
$$

Note that $\beta_{4}$ does not contribute to the background dynamics. A prime denotes the derivative of any quantities $X$ with respect to $\alpha . X^{\prime}$ is then related to its derivative with respect to $t$ by $X^{\prime}=(d X / d t) H^{-1}=\dot{X} H^{-1}$ where $H=d \alpha / d t=\dot{\alpha}$ is the Hubble parameter. From Eqs. 
(11) and (12), one obtains the energy equation for the vector field $u$

$$
\rho_{u}^{\prime}+3\left(\rho_{u}+p_{u}\right)=+3 H^{2} \beta^{\prime},
$$

and for the scalar field

$$
\rho_{\phi}^{\prime}+3\left(\rho_{\phi}+p_{\phi}\right)=-3 H^{2} \beta^{\prime} .
$$

The total energy equation in the presence of both the vector and the scalar fields is, accordingly,

$$
\rho^{\prime}+3(\rho+p)=0, \quad \rho=\rho_{u}+\rho_{\phi} .
$$

Substituting Eq. (10) into the Einstein equations (8), we obtain two independent equations, called the Friedmann equations, as follows:

$$
\begin{aligned}
\left(1+\frac{1}{8 \pi G \beta}\right) H^{2}= & \frac{1}{3 \beta} \rho_{\phi} \\
\left(1+\frac{1}{8 \pi G \beta}\right)\left(H H^{\prime}+H^{2}\right)= & -\frac{1}{6}\left(\frac{\rho_{\phi}}{\beta}+\frac{3 p_{\phi}}{\beta}\right) \\
& -H^{2} \frac{\beta^{\prime}}{\beta} .
\end{aligned}
$$

The second term on RHS of Eq. (18) is a consequence of the coupling vector field as a function of scalar field. If $\beta_{i}=0$, thus without the vector field, the above equations reduce to the conventional ones. And in the case $\beta_{i}=$ const., the above equations lead to the Friedmann equations given in Ref. [3].

Let us define the effective coupling function as follows

$$
\bar{\beta} \equiv \beta+\frac{1}{8 \pi G},
$$

then Eqs. (17) and (18) can be rewritten as

$$
\begin{aligned}
H^{2} & =\frac{1}{3 \bar{\beta}} \rho_{\phi}, \\
\frac{H^{\prime}}{H} & =-\frac{\bar{\beta}^{\prime}}{\bar{\beta}}-\frac{3}{2} \gamma_{\phi} .
\end{aligned}
$$

where the equation of state for the scalar field is defined by $\gamma_{\phi}=1+p_{\phi} / \rho_{\phi}$.

For a given scalar field Lagrangian with the FRW background, we can obtain the equations of motion for a scalar field by using Eq. (15). Let us consider the Lagrangian density of a scalar field $\phi$ with a potential $V(\phi)$ in Eq. (1):

$$
\mathcal{L}_{\phi}=-\frac{1}{2}(\nabla \phi)^{2}-V(\phi)
$$

where $(\nabla \phi)^{2}=g^{\mu \nu} \partial_{\mu} \phi \partial_{\nu} \phi$. For the homogeneous field, the energy density $\rho_{\phi}$ and the pressure $p_{\phi}$ of the scalar field may be found as follows

$$
\begin{aligned}
\frac{\rho_{\phi}}{V} & =\left(\frac{6 \bar{\beta}}{6 \bar{\beta}-\phi^{\prime 2}}\right), \\
\frac{p_{\phi}}{V} & =-2\left(\frac{3 \bar{\beta}-\phi^{\prime 2}}{6 \bar{\beta}-\phi^{\prime 2}}\right), \\
\gamma_{\phi} & =\frac{\phi^{\prime 2}}{3 \bar{\beta}} .
\end{aligned}
$$

Substituting Eq. (23) into Eq. (20) and also substituting Eq. (25) into Eq. (21), the Friedmann equations lead to

$$
\begin{aligned}
& H^{2}\left(1-\frac{\phi^{\prime 2}}{6 \bar{\beta}}\right)=\frac{1}{3 \bar{\beta}} V, \\
& \frac{H^{\prime}}{H}=-\frac{\bar{\beta}^{\prime}}{\bar{\beta}}-\frac{1}{2} \frac{\phi^{\prime 2}}{\bar{\beta}} .
\end{aligned}
$$

Now differentiating Eq. (23) with respect to $\alpha$ and using Eq. (15), we obtain a dynamical equation for the scalar field $\phi$,

$$
\phi^{\prime \prime}=-\left(\frac{H^{\prime}}{H}+3\right) \phi^{\prime}-\frac{V_{, \phi}}{H^{2}}-3 \bar{\beta}_{, \phi},
$$

which is subject to the Friedmann constraint given by Eq. (26).

\section{ATTRACTOR SOLUTIONS FOR PURELY SCALAR FIELD}

Equations (26)-(28) are the governing equations of motion which we will use to study dynamical attractor for purely scalar field. We introduce the following dimensionless variables [25]:

$$
\begin{array}{rlrl}
x^{2} & \equiv \frac{\phi^{\prime 2}}{6 \bar{\beta}}, & y^{2} \equiv \frac{V}{3 H^{2} \bar{\beta}}, \\
\lambda_{1} \equiv-\frac{\bar{\beta}_{, \phi}}{\sqrt{\bar{\beta}},} & \lambda_{2} \equiv-\sqrt{\bar{\beta}} \frac{V_{, \phi}}{V}, \\
\Gamma_{1} \equiv \frac{\bar{\beta} \bar{\beta}, \phi \phi}{\bar{\beta}_{, \phi}^{2}}, & \Gamma_{2} \equiv \frac{V V_{, \phi \phi}}{V_{, \phi}^{2}}+\frac{1}{2} \frac{\bar{\beta}_{, \phi} / \bar{\beta}}{V_{, \phi} / V},
\end{array}
$$

and, accordingly, the governing equations of motion could be reexpressed as the following system of equations:

$$
\begin{aligned}
& x^{\prime}=-3 x\left(1-x^{2}\right)+\sqrt{\frac{3}{2}}\left(\lambda_{1}+\lambda_{2}\right) y^{2}, \\
& y^{\prime}=\left[3 x-\sqrt{\frac{3}{2}}\left(\lambda_{1}+\lambda_{2}\right)\right] x y \\
& \lambda_{1}^{\prime}=-\sqrt{6} \lambda_{1}^{2}\left(\Gamma_{1}-\frac{1}{2}\right) x \\
& \lambda_{2}^{\prime}=-\sqrt{6} \lambda_{2}^{2}\left(\Gamma_{2}-1\right) x
\end{aligned}
$$

where a prime denotes a derivative with respect to the logarithm of the scale factor, $\alpha=\ln a$.

In general, the parameters $\lambda_{1}, \lambda_{2}, \Gamma_{1}$ and $\Gamma_{2}$ are variables dependent on $\phi$ and completely associated with the Lorentz violation. In particular, $\lambda_{1}$ and $\Gamma_{1}$ are purely Lorentz violation parameters and $\Gamma_{1}$ can be written as a function of $\lambda_{1}$ in this case. By definition (31), $\Gamma_{2}$ can be written as a function of $\lambda_{1}$ and $\lambda_{2}$. Thus, in order to construct viable Lorentz violation model, we require that the coupling function $\bar{\beta}$ and the potential function $V$ should satisfy the condition $\Gamma_{1}>1 / 2$ and $\Gamma_{2}>1$, 
respectively. In this paper, we want to discuss the phase space, then we need certain constraints on the coupling function and potential function. In particular, we study the case of constants $\Gamma_{1}$ and $\Gamma_{2}$. We take the form of $\Gamma_{1}$ and $\Gamma_{2}$ as $\Gamma_{1}=1 / 2$ and $\Gamma_{2}=1$, respectively. Equations (34) and (35) imply that $\lambda_{1}$ and $\lambda_{2}$ are nearly constants in this case.

Integrating Eq. (31) with respect to $\phi$, we obtain a Lorentz violating model

$$
\bar{\beta}(\phi)=\xi \phi^{2}, \quad V(\phi)=V_{0} \phi^{n},
$$

where $\xi, n$ and $V_{0}$ are parameters. Hence,

$$
\lambda_{1}=-2 \sqrt{\xi}, \quad \lambda_{2}=\frac{n}{2} \lambda_{1} .
$$

In the following, we study the case power-law potential $n>0$.

From the above equation, we require $\xi>0$. Then, the dynamical system of equations (32)-(35) can be rewritten as an autonomous system:

$$
\begin{aligned}
& x^{\prime}=-3 x\left(1-x^{2}\right)-(n+2) \sqrt{\frac{3 \xi}{2}} y^{2}, \\
& y^{\prime}=\left[3 x+(n+2) \sqrt{\frac{3 \xi}{2}}\right] x y .
\end{aligned}
$$

The equation (26) leads to the constraint

$$
x^{2}+y^{2}=1 .
$$

In term of the new variable, the equation (27) reads

$$
\frac{H^{\prime}}{H}=-3 x^{2}-2 \sqrt{6 \xi} x .
$$

Integrating this equation with respect to $\alpha$ one shows that all critical points, where $x$ is a non-zero constant, correspond to an evolution of the Hubble parameter $H$ given by $H \propto e^{-\alpha / p}$, where

$$
p \equiv \frac{1}{3 x^{2}+2 \sqrt{6 \xi} x} .
$$

An inflationary phase is one wherein the universe undergoes an accelerating expansion, i.e., the scale factor satisfies $\ddot{a}>0$. Inflation ends when this condition is violated. In the frame of Lorentz violating scalar-vectortensor, the condition for the accelerating universe is

$$
\sqrt{\xi} x<\frac{1}{6} \sqrt{\frac{3}{2}}\left(1-\frac{3}{2} \gamma_{\phi}\right),
$$

where $\gamma_{\phi}$ is an equation of state for the scalar field, $\gamma_{\phi}=1+\omega_{\phi}$. When the condition (43) is satisfied, we find three types of the inflationary solution given by Eq. (42): the power-law inflation $(p>0)$, the de Sitter inflation $(1 / p=0)$ and the superinflation $(p=-|p|<0)$, depending on the values of $\xi$. We will show that there

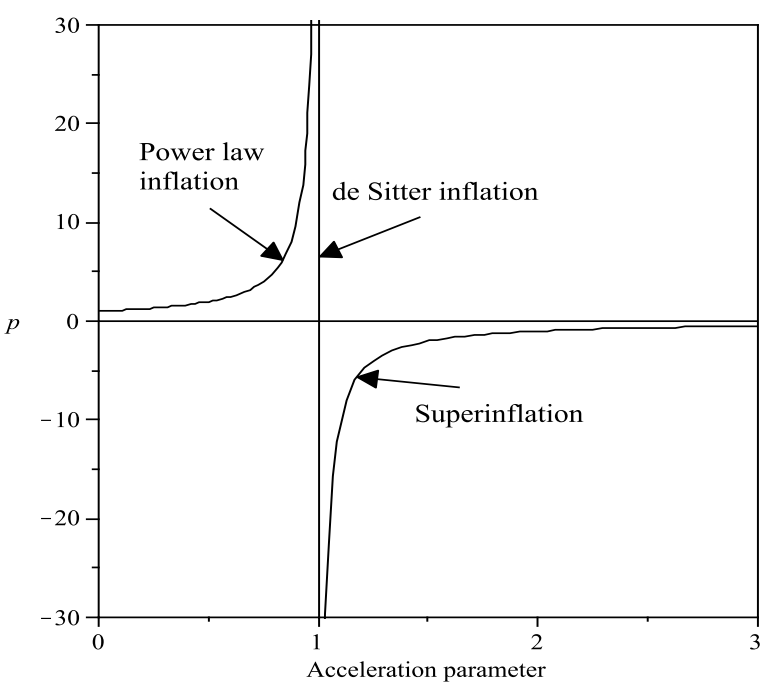

FIG. 1: There are three possibilities of the inflationary solutions in the Lorentz violating scalar-vector-tensor theory: the power-law inflation, the de Sitter inflation and the superinflation.

exists a stable attractor for this three types of the inflationary solution in the framework of Lorentz violating scalar-vector-tensor theory of gravity. In the conventional case, $\xi \rightarrow 0$, we are only left with a power-law inflation $p=1 / 3 x^{2}$, where the condition for the accelerated universe is, accordingly, $\gamma_{\phi}<2 / 3$. In Fig. 1, we show three possibilities of the inflationary solutions. Here we define an acceleration parameter,

$$
\bar{q} \equiv 1+\frac{\ddot{\alpha}}{\dot{\alpha}^{2}}=1-\frac{3}{2} \gamma_{\phi}-2 \sqrt{6 \xi},
$$

that simplifies

$$
p=\frac{1}{1-\bar{q}} .
$$

Then, the condition for the accelerating universe (43) becomes $\bar{q}>0$. It can be shown that the inflationary (accelerated) solutions are classified into three cases (see Fig. 10: a) $0<\bar{q}<1$, a solution in which $p>0$. In this case we have a power law inflation where $a(t) \sim t^{p}$. b) $\bar{q}=1,1 / p=0$. This is a de Sitter solution $a(t) \sim$ $e^{H t}$. c) $\bar{q}>1$. In this case, $p \equiv-|p|<0$. Hence, the solution becomes $a(t) \sim(-t)^{-|p|}, t<0$. Thus, this solution represents the super-inflationary universe.

In order to study the stability of the critical points, using the constraint Eq. (66), we first reduce Eqs. (38) and (39) to a one dimensional equation,

$$
x^{\prime}=-3\left[x+(n+2) \sqrt{\frac{\xi}{6}}\right]\left(1-x^{2}\right) .
$$

If we linearize the system about the critical points $x \rightarrow$ $x_{c}+u$, we could readily write the first-order perturbation 
equation as

$$
u^{\prime}=\left[2 x\left(3 x+(n+2) \sqrt{\frac{3 \xi}{2}}\right)-3\left(1-x^{2}\right)\right] u,
$$

which yields one eigenvalue.

Depending on the values of $\xi$ and $n$, we find three critical points where $x^{\prime}$ and $y^{\prime}$ vanish:

- Point $\left(A_{ \pm}\right)$: $\left(x_{c}, y_{c}\right)=( \pm 1,0)$ is a kinetic dominated solution.

- Point $(B)$ : $\left(x_{c}, y_{c}\right)=\left(-(n+2) \sqrt{\xi / 6}, \sqrt{1-(n+2)^{2} \xi / 6}\right)$ is a potential-kinetic dominated solution.

Notice that the above critical points depend on the values of the effective coupling parameter $\xi$ and the power of the potential $n$. For the positive values of $\xi$ and $n$, the linear perturbation shows that the point $\left(A_{+}\right)$is always unstable, whereas the point $\left(A_{-}\right)$is stable for $\xi>6 /(n+2)^{2}$. Thus, we only left with one possibility attractor solution in the kinetic dominated solution. The potential-kinetic dominated solution, point $(B)$, is stable for $0<\xi<6 /(n+2)^{2}$. In this case, the point $\left(A_{-}\right)$is unstable.

For the point $\left(A_{-}\right)$, the universe accelerates for $\xi>$ $1 / 6$. Combining the stability of the critical point and the condition for the accelerating universe, the inflationary attractor solutions are given by $0<n \leq 4, \xi>6 /(n+2)^{2}$ and $n>4, \xi>1 / 6$. Then, we find that the three types of inflationary (accelerated) solutions depend on the potential parameter and the effective coupling parameter.

- The power-law inflationary attractor:

$$
\begin{array}{ll}
2<n \leq 4, & \frac{6}{(n+2)^{2}}<\xi<\frac{3}{8} \\
n>4, & 1 / 6<\xi<3 / 8 .
\end{array}
$$

- The de Sitter inflationary attractor:

$$
n>2, \quad \xi=\frac{3}{8} .
$$

- The super-inflationary attractor:

$$
\begin{array}{ll}
0<n \leq 2, & \xi>\frac{6}{(n+2)^{2}}, \\
n>2, & \xi>\frac{3}{8} .
\end{array}
$$

In the case potential-kinetic dominated solution, the accelerated expansions of the universe are given by $0<$ $n \leq 2, \xi>0$ and $n>2,0<\xi<2 /\left(n^{2}-4\right)$. Then we find

- The power-law inflationary attractor:

$$
\begin{array}{cl}
2<n \leq 4 \quad, & 0<\xi<\frac{6}{(n+2)^{2}}, \\
n>4 \quad, & 0<\xi<\frac{2}{\left(n^{2}-4\right)} .
\end{array}
$$

- The de Sitter inflationary attractor:

$$
n=2, \quad 0<\xi<3 / 8 .
$$

- The super-inflationary attractor:

$$
0<n<2, \quad 0<\xi<\frac{6}{(n+2)^{2}} .
$$

In summary, the effective coupling parameter and the potential model determine the stabilities of the critical points and the inflationary solutions. There are two possibilities of attractor solutions given by the critical point $\left(A_{-}\right)$and $(B)$.

\section{ATTRACTOR SOLUTIONS IN THE PRESENCE OF BAROTROPIC FLUID}

For a realistic model, we consider the effect of an additional component. We carry out cosmological dynamics of the scalar field $\phi$ in the presence of a barotropic fluid whose equation of state is given by $p_{\gamma}=(\gamma-1) \rho_{\gamma}$, where $\gamma$ is an adiabatic index, $0 \leq \gamma \leq 2$. We assume that there is a barotropic fluid, not explicitly coupled to the scalar field and the vector field. Then, the total energy-momentum tensor in Eq. (8) for this case is $T_{\mu \nu}=T_{\mu \nu}^{(u)}+T_{\mu \nu}^{(\phi)}+T_{\mu \nu}^{(\gamma)}$, where

$$
T_{\mu \nu}^{(\gamma)}=\left(\rho_{\gamma}+p_{\gamma}\right) n_{\mu} n_{\nu}+p_{\gamma} g_{\mu \nu}
$$

is the energy-momentum tensor of the matter field. Here $n^{\mu}$ is the four velocity. The time and space components of the Einstein equations (8) yield

$$
3 H^{2}=8 \pi G\left(\rho_{u}+\rho_{\phi}+\rho_{\gamma}\right),
$$

and

$$
2 H^{\prime} H=-8 \pi G\left(\rho_{u}+p_{u}+\rho_{\phi}+p_{\phi}+\rho_{\gamma}+p_{\gamma}\right) .
$$

Substituting equations (11) and (23) for the energy density of vector field and scalar field into equation (58), respectively, one finds

$$
H^{2}=\frac{1}{3 \bar{\beta}}\left(\frac{V+\rho_{\gamma}}{1-\frac{\phi^{\prime 2}}{6 \bar{\beta}}}\right) .
$$

The evolution equation for a barotropic fluid is

$$
\rho_{\gamma}^{\prime}=-3 \gamma \rho_{\gamma}
$$

The scalar field obeys the same equation of motion, Eq. (28). The second Friedmann equation, Eq. (59), becomes

$$
\frac{H^{\prime}}{H}=-\frac{\bar{\beta}^{\prime}}{\bar{\beta}}-\frac{1}{2} \frac{\phi^{\prime 2}}{\bar{\beta}}-\gamma \frac{\rho_{\gamma}}{2 H^{2} \bar{\beta}} .
$$

Equation (28) together with Eqs. (60)- 62) are the governing equations of motion which will be used to study 
dynamical attractor for a scalar field in the presence of the barotropic fluid.

For the cases of the constants $\lambda_{1}$ and $\lambda_{2}$, the governing equations can be written as the two-dimensional autonomous system:

$$
\begin{aligned}
x^{\prime}= & \frac{3}{2} x\left[\gamma\left(1-x^{2}-y^{2}\right)+2 x^{2}\right]-3 x \\
& +\sqrt{\frac{3}{2}}\left[\lambda_{1}\left(1-x^{2}\right)+\lambda_{2} y^{2}\right] \\
y^{\prime}= & \frac{3}{2} y\left[\gamma\left(1-x^{2}-y^{2}\right)+2 x^{2}\right] \\
& -\sqrt{\frac{3}{2}}\left(\lambda_{1}+\lambda_{2}\right) x y .
\end{aligned}
$$

The system of equations are symmetric under the reflection $(x, y) \rightarrow(x,-y)$. In what follows, we will restrict our discussion to the existence and stability of critical points to the upper half plane $y \geq 0$. In the case $\beta_{i}=$ const., $\lambda_{1} \rightarrow 0$, the scalar field dynamics in the Lorentz violating scalar-vector-tensor theories is then reduced to the scalar field dynamics in the conventional one. But, the effective gravitational constant is rescaled by Eq. (19). In this case, the cosmological attractor solutions can be studied by a scalar exponential potential of the form $V(\phi)=V_{0} \exp \left(-\lambda_{2} \phi / \sqrt{\bar{\beta}}\right)$ where $\bar{\beta}=$ const. This exponential potential gives rise to scaling solutions for the scalar field [17].

Also, the Friedmann constraint, Eq. (60), becomes

$$
\Omega_{\phi}+\frac{\rho_{\gamma}}{3 H^{2} \bar{\beta}}=1
$$

where the contribution of the scalar field to the total energy density is

$$
\Omega_{\phi}=\frac{\rho_{\phi}}{3 H^{2} \bar{\beta}}=x^{2}+y^{2} .
$$

In term of the new variable we find

$$
\frac{H^{\prime}}{H}=-\frac{3}{2} \gamma_{e f f},
$$

where we have defined the effective equation of state of the universe:

$$
\gamma_{e f f} \equiv \gamma+\Omega_{\phi}\left(\gamma_{\phi}-\gamma\right)-2 \sqrt{\frac{2}{3}} \lambda_{1} x
$$

When $x$ is a non zero constant, Eq. (67) corresponds to an evolution of the Hubble parameter given by

$$
H \propto e^{-\alpha / p},
$$

where

$$
p \equiv \frac{1}{\frac{3}{2} \gamma_{e f f}} .
$$

In the presence of barotropic fluid, the condition for the accelerating universe is

$$
\lambda_{1} x>\frac{1}{2} \sqrt{\frac{3}{2}}\left(\gamma+\Omega_{\phi}\left(\gamma_{\phi}-\gamma\right)-\frac{2}{3}\right),
$$

The equation (70) can be simplified by

$$
p=\frac{1}{1-\bar{q}},
$$

where we have defined an acceleration parameter $\bar{q}$,

$$
\bar{q}=1-\frac{3}{2} \gamma_{e f f} .
$$

Thus the three types of inflation are also possible in the presence of the barotropic fluid.

\section{A. Stability of the fixed points}

The critical points $\left(x_{c}, y_{c}\right)$ are obtained by imposing the conditions $x^{\prime}=0$ and $y^{\prime}=0$. Substituting linear perturbation $x \rightarrow x_{c}+u$ and $y \rightarrow y_{c}+v$ about the critical points into Eqs. (63) and (64), we obtain, to first-order in the perturbation, the equations of motion

$$
\left(\begin{array}{l}
u^{\prime} \\
v^{\prime}
\end{array}\right)=M\left(\begin{array}{l}
u \\
v
\end{array}\right)
$$

In what follows we clarify the properties of the five critical points given in Table \(see Appendix). We analyze the stability of the critical points with the background barotropic fluid $0 \leq \gamma \leq 2$. The stable solutions of these critical points are

a) Two kinetic dominated solutions

For the point $\left(A_{+}\right)$, the eigenvalues are

$$
m_{1}=3+(n+2) \sqrt{\frac{3 \xi}{2}}, \quad m_{2}=3(2-\gamma)+2 \sqrt{6 \xi} .
$$

Thus, the critical point $\left(A_{+}\right)$is always unstable for $n>0$.

For the critical point $\left(A_{-}\right)$, the eigenvalues are

$$
m_{1}=3-(n+2) \sqrt{\frac{3 \xi}{2}}, \quad m_{2}=3(2-\gamma)-2 \sqrt{6 \xi} .
$$

The stable solutions for the kinetic dominated solution $\left(A_{-}\right)$are

$$
0 \leq \gamma \leq \frac{2 n}{n+2}, \quad \xi>\frac{3(2-\gamma)^{2}}{8},
$$

and

$$
\frac{2 n}{n+2}<\gamma \leq 2, \quad \xi>\frac{6}{(n+2)^{2}} .
$$


In this kinetic dominated solution, we have the following relations:

$$
\begin{aligned}
& \Omega_{\phi}=1, \quad \gamma_{\phi}=2, \quad \gamma_{e f f}=2+4 \sqrt{\frac{2 \xi}{3}}, \\
& \frac{1}{p_{A}}=1-\bar{q}_{A}, \quad \bar{q}_{A}=-2+2 \sqrt{6 \xi},
\end{aligned}
$$

b) Scalar field dominated solution, point $(B)$

The eigenvalues are

$$
m_{1}=-3+\frac{(n+2)^{2}}{2} \xi, \quad m_{2}=-3 \gamma+n(n+2) \xi .
$$

The stable solutions for the scalar field dominated solution are

$$
0<\gamma<\frac{2 n}{n+2}, \quad 0<\xi<\frac{3 \gamma}{n(n+2)},
$$

and

$$
\frac{2 n}{n+2} \leq \gamma \leq 2, \quad 0<\xi<\frac{6}{(n+2)^{2}} .
$$

The critical point exists for $0<\xi<6 /(n+2)^{2}$. In this case, we find the following relations:

$$
\begin{aligned}
& \Omega_{\phi}=1, \gamma_{\phi}=\frac{1}{3}(n+2)^{2} \xi, \gamma_{e f f}=\frac{1}{3}\left(n^{2}-4\right) \xi \\
& \frac{1}{p_{B}}=1-\bar{q}_{B}, \quad \bar{q}_{B}=1-\frac{1}{2}\left(n^{2}-4\right) \xi
\end{aligned}
$$

The universe accelerates for $0<n \leq 2, \xi>0$ and $n>2$, $0<\xi<2 /\left(n^{2}-4\right)$.

c) Kinetic scaling solution, point $(C)$

The eigenvalues are

$$
m_{1}=\frac{3 \gamma}{2}-\frac{2 n \xi}{2-\gamma}, \quad m_{2}=-\frac{3(2-\gamma)}{2}+\frac{4 \xi}{(2-\gamma)} .
$$

The stable solutions for the kinetic scaling solution are

$$
0 \leq \gamma<\frac{2 n}{n+2}, \quad \frac{3 \gamma(2-\gamma)}{4 n}<\xi<\frac{3(2-\gamma)^{2}}{8} .
$$

We obtain the following relations:

$$
\begin{aligned}
& \Omega_{\phi}=\frac{8 \xi}{3(2-\gamma)^{2}}, \\
& \gamma_{\phi}=2, \quad \gamma_{e f f}=\gamma-\frac{8 \xi}{3(2-\gamma)}, \\
& \frac{1}{p_{C}}=1-\bar{q}_{C}, \quad \bar{q}_{C}=1-\frac{3 \gamma}{2}+\frac{4 \xi}{(2-\gamma)} .
\end{aligned}
$$

The universe accelerates for $0 \leq \gamma \leq 2 / 3, \xi>0$ and $2 / 3<\gamma<2, \xi>(3 \gamma-2)(2-\gamma) / 8$.
Note that the inflationary attractor solution dose not exist for $\gamma=4 / 3$. However, in the background matter $(\gamma=1)$, the de Sitter inflationary attractor corresponds to the parameters, $n>3,0<\xi$. the superinflationary attractor does not exist in this case and the powerlaw inflationary attractor corresponds to the parameters, $2<n \leq 6,3 / 4 n<\xi<3 / 8$ and $n>6,1 / 8<\xi<3 / 8$.

d) Scalar field scaling solution, point $(D)$

The eigenvalues are

$$
m_{1,2}=\frac{T}{2}\left(1 \pm \sqrt{1-\frac{4 D}{T^{2}}}\right)
$$

where

$$
\begin{aligned}
& T=-3\left(1-\frac{(n+2) \gamma}{2 n}\right) \\
& D=\frac{3[4 n \xi-3 \gamma(2-\gamma)][3 \gamma-(n+2) n \xi]}{2 n^{2} \xi} .
\end{aligned}
$$

The stable solutions for the scalar field scaling solution are given by

$$
0<\gamma<\frac{2 n}{n+2}, \quad \frac{3 \gamma}{n(n+2)} \leq \xi \leq \frac{3 \gamma(2-\gamma)}{4 n} .
$$

The critical point exists for $0<\gamma<2,0<\xi<3 \gamma(2-$ $\gamma) / 4 n$. We find the following relations:

$$
\begin{aligned}
& \Omega_{\phi}=\frac{3 \gamma}{n^{2} \xi}\left(1-\frac{2 n \xi}{3 \gamma}\right), \\
& \gamma_{\phi}=\frac{3 \gamma^{2}}{3 \gamma-2 n \xi}, \quad \gamma_{e f f}=\frac{\gamma(n-2)}{n}, \\
& \frac{1}{p_{D}}=1-\bar{q}_{D}, \quad \bar{q}_{D}=1-\frac{3 \gamma(n-2)}{2 n},
\end{aligned}
$$

and the universe accelerates for $0<n<3,0<\gamma<2$ and $n \geq 3,0<\gamma<2 n / 3(n-2)$.

From the above analyzes of the critical points, when one of the critical point is stable, then the other critical points are unstable or saddle depending on the values of $\gamma$ and $\xi$.

\section{B. Phase-space diagrams}

In this subsection, we show phase-space diagrams of the stability solutions. Fig. 2 shows that different regions in the $(\gamma, \xi)$ parameter space lead to different qualitative evolutions for the potential model $V(\phi)=V_{0} \phi^{4}$.

- Region A

$$
0<\gamma \leq 4 / 3, \quad \xi>3(2-\gamma)^{2} / 8,
$$

and

$$
4 / 3<\gamma<2, \quad \xi>1 / 6 .
$$




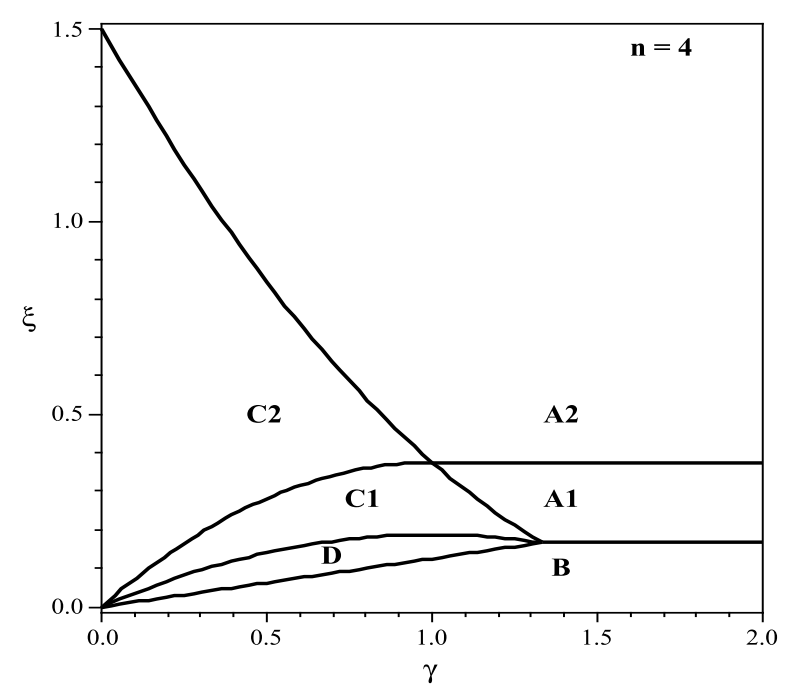

FIG. 2: Region of $(\gamma, \xi)$ parameter space for the potential model $V(\phi)=V_{0} \phi^{4}$.

The kinetic dominated solution $\left(A_{-}\right)$is stable in this case. The scalar field dominated solution and the kinetic scaling solution are saddle for $\xi>1 / 6$ and $\xi>3(2-\gamma)^{2} / 8$, respectively. The scalar field scaling solution is unstable. The effective equation of state is, accordingly, $\gamma_{e f f}=2-4(2 \xi / 3)^{1 / 2}$ and the universe accelerates for $\xi>1 / 6$. For the inflationary attractor solutions, we obtain: i) The power-law inflationary attractor solution (Region A1) corresponds to region of $(\gamma, \xi)$ parameter space: $1<\gamma<4 / 3,3(2-\gamma)^{2} / 8<\xi<3 / 8$ and $4 / 3 \leq \gamma \leq 2,1 / 6<\xi<3 / 8$, ii) The de Sitter inflationary attractor solution (Line $\xi=3 / 8$ ) corresponds to region of $(\gamma, \xi)$ parameter space: $1<\gamma<2, \xi=3 / 8$, and iii) The superinflationary attractor solution (Region A2) correspond to region of $(\gamma, \xi)$ parameter space: $0 \leq \gamma \leq 1$, $\xi>3(2-\gamma)^{2} / 8$ and $1<\gamma \leq 2, \xi>3 / 8$. In Fig. 3, we show the phase plane plot for $\gamma=1$ and $\xi=1$. The superinflationary attractor is the kinetic dominated solution.

- Region B

$$
0<\gamma \leq 4 / 3, \quad 0<\xi<\frac{\gamma}{8}
$$

and

$$
4 / 3<\gamma \leq 2, \quad 0<\xi<1 / 6 .
$$

The scalar field dominated solution is stable in this case and it exists for $\xi<1 / 6$. The kinetic dominated solution and the scalar field scaling solution are saddle for $0<\xi<1 / 6$ and $0<\xi<\gamma / 8$, respectively. The kinetic scaling solution is unstable. The universe accelerates for $0<\xi<1 / 6$. We only find the power-law inflationary attractor solution in this case, and it corresponds to region of $(\gamma, \xi)$ parameter space: $0<\gamma \leq 4 / 3,0<\xi<\gamma / 8$ and $4 / 3<\gamma \leq 2,0<\xi<1 / 6$. In Fig. 4, we show the phase plane plot for $\gamma=1$ and $\xi=1 / 16$. The power-law inflationary attractor is the scalar field dominated solution.

- Region C

$0 \leq \gamma<\frac{4}{3}, \quad \frac{3 \gamma(2-\gamma)}{16}<\xi<\frac{3(2-\gamma)^{2}}{8}$.

The kinetic scaling solution is stable in this case. The kinetic dominated solution, the scalar field dominated solution, and the scalar field scaling solution are saddle for $\xi<3(2-\gamma)^{2} / 8, \xi>1 / 6$, and $3 \gamma(2-\gamma) / 16<\xi$, respectively. Also, for the inflationary solutions, there are three cases to be considered, i.e., i) The power-law inflationary attractor solution (Region C1) corresponds to region of $(\gamma, \xi)$ parameter space: $0<\gamma \leq 1$, $3 \gamma(2-\gamma) / 16<\xi<3 \gamma(2-\gamma) / 8$ and $1<\gamma<4 / 3$, $3 \gamma(2-\gamma) / 16<\xi<3(2-\gamma)^{2} / 8$, ii) The de Sitter inflationary attractor solution (Line $\xi=3 \gamma(2-\gamma) / 8$ ) corresponds to region of $(\gamma, \xi)$ parameter space: $0<\gamma<1, \xi=3 \gamma(2-\gamma) / 8$, and iii) The superinflationary attractor solution (Region C2) corresponds to region of $(\gamma, \xi)$ parameter space: $0 \leq \gamma<1$,

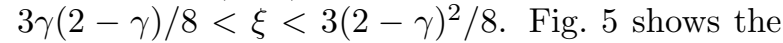
phase plane plot for $\gamma=1$ and $\xi=1 / 4$. The powerlaw inflationary attractor is the kinetic scaling solution.

- Region D

The scalar field scaling solution exists for $0<\gamma<$ $2,0<\xi<3 \gamma(2-\gamma) / 16$, and it is stable for

$$
0<\gamma<\frac{4}{3}, \quad \frac{\gamma}{8}<\xi<\frac{3 \gamma(2-\gamma)}{16} .
$$

The kinetic dominated solution is unstable. The scalar field dominated solution and the kinetic scaling solution are saddle for $\xi>\gamma / 8$ and $\xi<$ $3 \gamma(2-\gamma) / 6$, respectively. The universe accelerates for $0 \leq \gamma<4 / 3$. For the inflationary attractor solutions, we only find the power-law inflationary attractor solution in this case, corresponding to the region of $(\gamma, \xi)$ parameter space: $0 \leq \gamma<4 / 3$, $\gamma / 8<\xi<3 \gamma(2-\gamma) / 16$. In Fig. 66, we show the phase plane plot for $\gamma=1$ and $\xi=2.5 / 16$. The power-law inflationary attractor is the scalar field scaling solution.

\section{Cosmological implications}

As we have shown in the previous section, there are some possibilities that lead to an accelerated expansion: the kinetic dominated solutions and the scalar field dominated solutions together with scaling solutions. The 


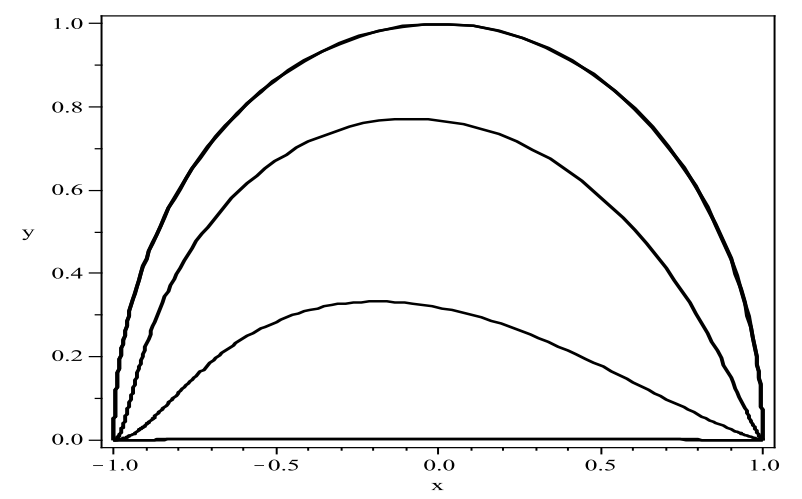

FIG. 3: The phase plane for the potential model $V=V_{0} \phi^{4}$, $\gamma=1$, and $\xi=1$. The attractor is a kinetic dominated solution with $x=-1, y=0$, where $\Omega_{\phi}=1, \gamma_{\phi}=2, \gamma_{\text {eff }}=$ $2(1-2 \sqrt{2 / 3})$ and $\bar{q}=2(\sqrt{6}-1)$.

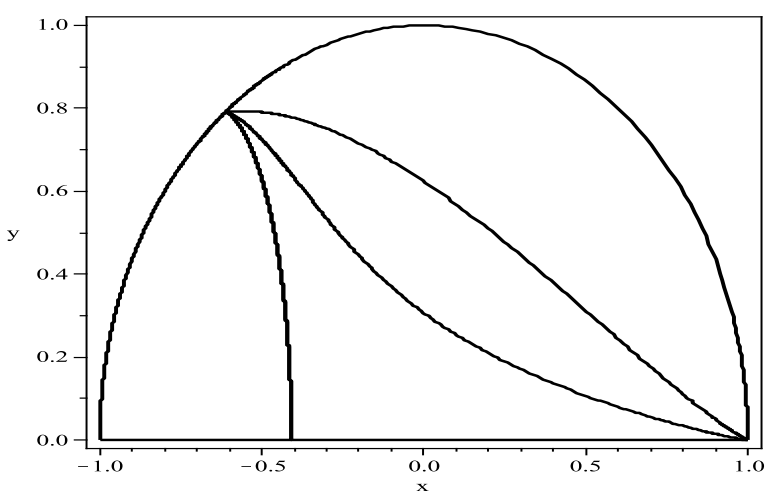

FIG. 4: The phase plane for the potential model $V=V_{0} \phi^{4}$, $\gamma=1$, and $\xi=1 / 16$. The attractor is a scalar field dominated solution with $x=-\sqrt{3 / 8}, y=\sqrt{5 / 8}$, where $\Omega_{\phi}=1, \gamma_{\phi}=$ $3 / 4, \gamma_{\text {eff }}=1 / 4$ and $\bar{q}=5 / 8$.

later, however, is not viable to explain an accelerated universe at the present epoch since $\Omega_{\phi} \simeq 0.65 \pm 0.05$ with $\omega_{\phi} \leq-0.4$ [27]. In this section we consider the cosmological implications of the scaling solutions. For the case power-law potential, we find two scaling solutions: the kinetic scaling solution and the scalar field scaling solution.

In the background radiation, we find the following relations for the kinetic scaling solution:

$$
\Omega_{\phi}=6 \xi, \quad \gamma_{\phi}=2, \quad \gamma_{e f f}=\frac{4}{3}(1-3 \xi) .
$$

The universe accelerates for $\xi>1 / 6$. However, this scaling solution is not viable to explain the accelerated universe in the background radiation, because the universe is unstable in this case.

The scalar field scaling solution has the density parameter,

$$
\Omega_{\phi}=\frac{4}{n^{2} \xi}\left(1-\frac{n \xi}{2}\right)
$$

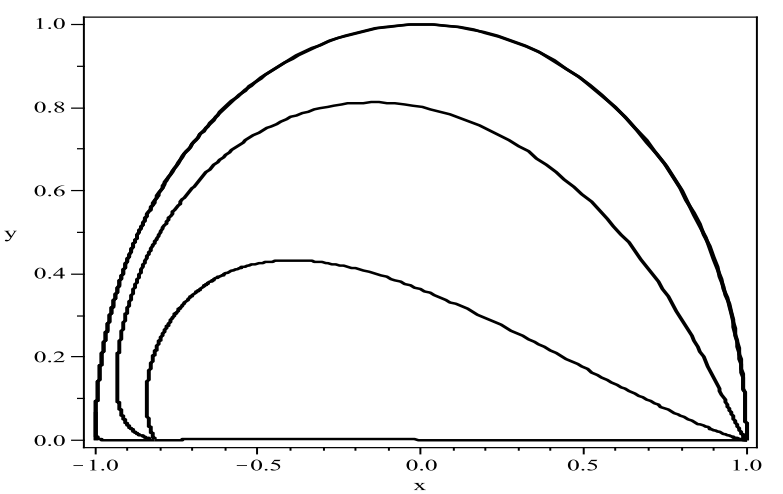

FIG. 5: The phase plane for the potential model $V=V_{0} \phi^{4}$, $\gamma=1$, and $\xi=1 / 4$. The attractor is a kinetic scaling solution with $x=-\sqrt{2 / 3}, y=0$, where $\Omega_{\phi}=2 / 3, \gamma_{\phi}=2, \gamma_{\text {eff }}=1 / 3$ and $\bar{q}=0.5$.

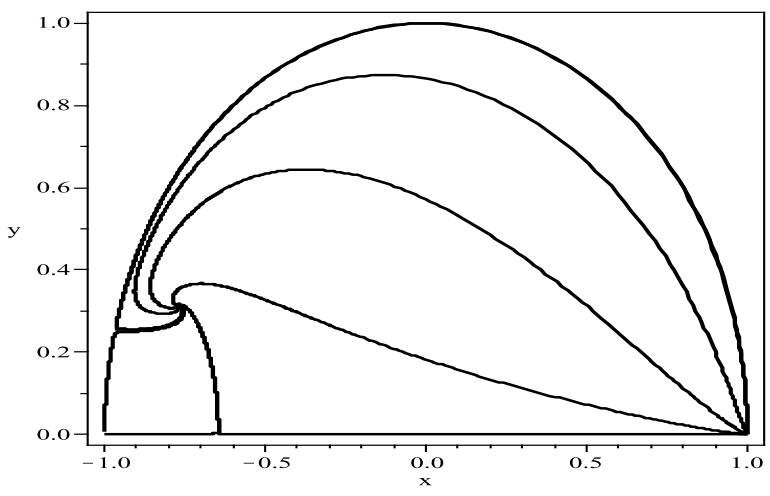

FIG. 6: The phase plane for the potential model $V=V_{0} \phi^{4}$, $\gamma=1$, and $\xi=2.5 / 16$. The scalar field dominated solution is the saddle at $x=-\sqrt{15} / 4, y=1 / 4$, and the attractor is a scalar field scaling solution with $x=-\sqrt{3 / 5}, y=1 / \sqrt{10}$, where $\Omega_{\phi}=7 / 10, \gamma_{\phi}=12 / 7, \gamma_{\text {eff }}=1 / 2$ and $\bar{q}=1 / 4$.

for $\gamma=4 / 3$, where ranges $\xi$ between $0<\xi<2 / 3 n$. We also obtain

$$
\gamma_{\phi}=\frac{8}{3(2-n \xi)}, \quad \gamma_{e f f}=\frac{4(n-2)}{3 n} .
$$

Moreover, using the standard nucleosynthesis and the observed abundances of primordial nuclides, the strong constraint is that the fractional energy density of scalar field at temperatures near $1 \mathrm{MeV}$ is $\Omega_{\phi}<\Omega_{\phi}^{\max }$. This implies

$$
\frac{4}{n\left(n \Omega_{\phi}^{\max }+2\right)}<\xi
$$

For $\Omega_{\phi}^{\max } \simeq 0.2$, the nucleosynthesis bound requires

$$
\frac{20}{n(n+10)}<\xi,
$$

for the Lorentz violation to be relevant. 


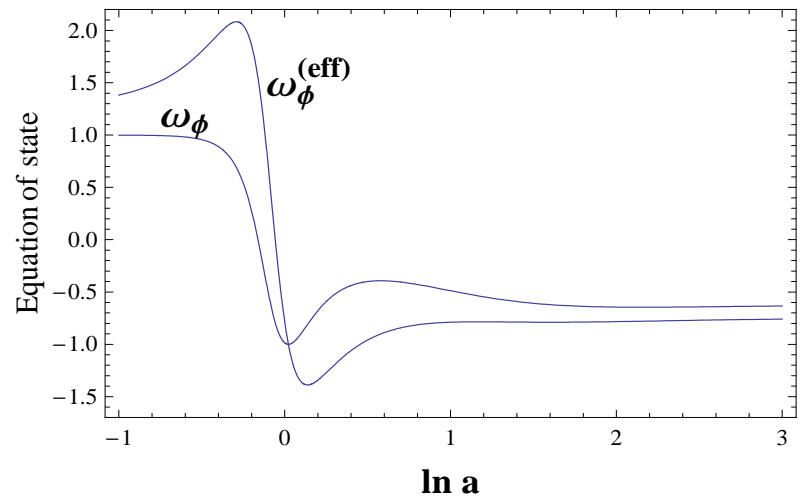

FIG. 7: The evolutions of the effective equation of state as a function of $\ln a$ for the case $\xi=1 / 32, \gamma=1$ and $n=4$. We choose the initial conditions $x_{i}=0.01, y_{i}=0.12$.

From Eq. (15), we define $\gamma_{\phi}^{(e f f)}=\gamma_{\phi}+H^{2} \bar{\beta}^{\prime} / \rho_{\phi}$ which is related with the effective equation of state parameter $\omega_{\phi}^{(e f f)}$ by the relationship $\omega_{\phi}^{(e f f)}=\omega_{\phi}+H^{2} \bar{\beta}^{\prime} / \rho_{\phi}$. In Fig. 7 we show the evolutions of equation of state as a function of $\alpha=\ln a$ for the case $\xi=1 / 32, \gamma=1$ and $n=4$. We choose the initial conditions $x_{i}=0.01$, $y_{i}=0.12$. The effective equation of state for the scalar field can cross -1 .

\section{CONCLUSIONS}

In this paper, we have studied the attractor solutions of the scalar field in the frame of Lorentz violating scalarvector-tensor theory of gravity. In this model, because of the dynamics of the effective coupling vector, the dynamics of the scalar field is modified from the conventional cosmology. The characteristic of the slope, $p$, and the condition of the accelerating universe are given by Eqs. (70) and (71), respectively.

For the model (36) without a barotropic fluid, the critical points of a dynamical system completely depend on the coupling parameter with typical potential. There exist two kinetic dominated solutions and one potentialkinetic dominated solution. Different regions in the $(\xi, p)$ parameter space lead to different qualitative evolutions. There are three typical inflationary solutions which are obtained from Eq. (42): the power-law inflation, the de Sitter inflation and the superinflation. Three types of inflationary attractor exist both in the kinetic dominated solution and scalar field dominated solution. Note that the superinflationary is stable in the case purely Lorentz violation without potential, where $\xi>3 / 2$.

We have extended the dynamical system of the evolution to a realistic universe model with a barotropic fluid. The stability of the critical point was discussed in Sec. IV. In this case, the fluid dominated solution is absent, instead, we find two scaling solutions: the kinetic scaling solution and the scalar field scaling solution. We have completely have shown the stable attractor in regions of $(\gamma, \xi)$ parameter space. Based on this parameter space, we have shown the phase plane plot for the four different qualitative evolutions, i.e. the kinetic dominated solution, the scalar field (potential-kinetic) dominated solution, the kinetic scaling solution and the scalar field scaling solution. Another result is that the scalar-vectortensor theory of gravity is still relevant at the nucleosynthesis. From this data, we derive the constraints for the value of the coupling parameter in Eqs. (107).

More interestingly, the model (36) allows for the inverse-power potential, $n<0$. As an example, in the case $n=-2$, we only have one attractor solution in the potential dominated solution. The effective equation of state parameter $\gamma_{\phi}$ is of the cosmological constant. Two attractor solutions, the scalar field dominated solution together with the scaling solution exist for $n<-2$.

When $\lambda_{1}$ is dynamically changing quantity, the effective coupling vector could track the evolution of the scalar field. There exists one stable critical point that gives an acceleration of the universe at late time with the equation of state parameter $\omega<-1 / 3$. Thus, we can have a dark energy scenario in the frame of scalar-vector-tensor theory of gravity [28]. Finally, we would like to emphasize that there exists an attractor solution in the Lorentz violating scalar-vector-tensor theory of gravity.

\section{Acknowledgments}

Arianto wishes to acknowledge all members of the Theoretical Physics Laboratory, the THEPI Divison of the Faculty of Mathematics and Natural Sciences, ITB, for the warmest hospitality. This research is financially supported by Riset Internasional ITB, No. 054/K01.7/PL/2008.

\section{APPENDIX: STABILITY OF THE CRITICAL POINTS FOR CONSTANTS $\lambda_{1}$ AND $\lambda_{2}$}

Let us consider the cases $\Gamma_{1}=1 / 2$ and $\Gamma_{2}=1$. From Eqs. (34)-(35), we obtain $\lambda_{1}$ and $\lambda_{2}$ nearly constants. The critical points $\left(x_{c}, y_{c}\right)$ are obtained by imposing the conditions $x^{\prime}=0$ and $y^{\prime}=0$. Substituting linear perturbation $x \rightarrow x_{c}+u$ and $y \rightarrow y_{c}+v$ about the critical points into Eqs. (63) and (64), to first-order in the perturbation, one obtains the equations of motion

$$
\left(\begin{array}{l}
u^{\prime} \\
v^{\prime}
\end{array}\right)=M\left(\begin{array}{l}
u \\
v
\end{array}\right),
$$


TABLE I: The properties of critical points with barotropic fluid in the Lorentz violating scalar-vector-tensor theory of gravity for constants $\lambda_{1}$ and $\lambda_{2}$.

\begin{tabular}{|c|c|c|c|c|}
\hline \hline Point & $x$ & $y$ & Existence & Stability \\
\hline$\left(A_{+}\right)$ & +1 & 0 & All $\lambda_{1}, \lambda_{2}$ and $\gamma$ & Stable: $\lambda_{1}>6-\sqrt{6} \lambda_{2} / \sqrt{6}$ and $\lambda_{1}>3(2-\gamma) / \sqrt{6}$ \\
\hline$\left(A_{-}\right)$ & -1 & 0 & All $\lambda_{1}, \lambda_{2}$ and $\gamma$ & Stable: $\lambda_{1}<-\left(6+\sqrt{6} \lambda_{2}\right) / \sqrt{6}$ and $\lambda_{1}<-3(2-\gamma) / \sqrt{6}$ \\
\hline$(B)$ & $\frac{1}{\sqrt{6}}\left(\lambda_{1}+\lambda_{2}\right)$ & {$\left[1-\frac{\left(\lambda_{1}+\lambda_{2}\right)^{2}}{6}\right]^{1 / 2}$} & $\left(\lambda_{1}+\lambda_{2}\right)^{2}<6$ & Stable: See Eq. (A.12) \\
\hline$(C)$ & $(2 / 3)^{1 / 2} \frac{\lambda_{1}}{(2-\gamma)}$ & 0 & All $\lambda_{2}, \gamma \neq 2$ and $\lambda_{1} \neq 0$ & Stable: See Eq. (A.15) \\
\hline$(D)$ & $(3 / 2)^{1 / 2} \frac{\gamma}{\lambda_{2}}$ & {$\left[\frac{3 \gamma(2-\gamma)}{2 \lambda_{2}^{2}}-\frac{\lambda_{1}}{\lambda_{2}}\right]^{1 / 2}$} & $\lambda_{1} \lambda_{2}<\frac{3 \gamma(2-\gamma)}{2}$ & Stable: See Eqs. (A.17)-(A.18) \\
\hline \hline
\end{tabular}

TABLE II: The scalar field density parameter $\Omega_{\phi}$, the equation of state $\gamma_{\phi}$, the effective equation of state $\gamma_{e f f}$, the slope $p$ and the acceleration parameter, $\bar{q}$, for the cases of constants $\lambda_{1}$ and $\lambda_{2}$.

\begin{tabular}{|c|c|c|c|c|c|}
\hline \hline Point & $\Omega_{\phi}$ & $\gamma_{\phi}$ & $\gamma_{\text {eff }}$ & $1 / p$ & $\bar{q}$ \\
\hline$\left(A_{+}\right)$ & 1 & 2 & $2-2 \sqrt{2 / 3} \lambda_{1}$ & $3-\sqrt{6} \lambda_{1}$ & $-2+\sqrt{6} \lambda_{1}$ \\
\hline$\left(A_{-}\right)$ & 1 & 2 & $2+2 \sqrt{2 / 3} \lambda_{1}$ & $3+\sqrt{6} \lambda_{1}$ & $-2-\sqrt{6} \lambda_{1}$ \\
\hline$(B)$ & 1 & $\frac{1}{3}\left(\lambda_{1}+\lambda_{2}\right)^{2}$ & $\frac{1}{3}\left(\lambda_{2}^{2}-\lambda_{1}^{2}\right)$ & $\frac{1}{2}\left(\lambda_{2}^{2}-\lambda_{1}^{2}\right)$ & $1-\frac{1}{2}\left(\lambda_{2}^{2}-\lambda_{1}^{2}\right)$ \\
\hline$(C)$ & $\frac{2 \lambda_{1}^{2}}{3(2-\gamma)^{2}}$ & 2 & $\gamma-\frac{2 \lambda_{1}^{2}}{3(2-\gamma)}$ & $\frac{3}{2} \gamma-\frac{\lambda_{1}^{2}}{(2-\gamma)}$ & $1-\frac{3}{2} \gamma+\frac{\lambda_{1}^{2}}{(2-\gamma)}$ \\
\hline$(D)$ & $\frac{3 \gamma}{\lambda_{2}^{2}}\left(1-\frac{\lambda_{1} \lambda_{2}}{3 \gamma}\right)$ & $\gamma\left(1-\frac{\lambda_{1} \lambda_{2}}{3 \gamma}\right)^{-1}$ & $\gamma\left(1-\frac{\lambda_{1}}{\lambda_{2}}\right)$ & $\frac{3}{2} \gamma\left(1-\frac{\lambda_{1}}{\lambda_{2}}\right)$ & $1-\frac{3}{2} \gamma\left(1-\frac{\lambda_{1}}{\lambda_{2}}\right)$ \\
\hline \hline
\end{tabular}

where the elements of the matrix $M$ are

$$
\begin{aligned}
M_{11}= & -3+3(2-\gamma) x^{2}+\frac{3}{2}\left[\gamma\left(1-x^{2}-y^{2}\right)+2 x^{2}\right] \\
& -\sqrt{6} \lambda_{1} x \\
M_{12}= & \left(\sqrt{6} \lambda_{2}-3 \gamma x\right) y \\
M_{21}= & {\left[3(2-\gamma) x-\sqrt{\frac{3}{2}}\left(\lambda_{1}+\lambda_{2}\right)\right] y } \\
M_{22}= & -3 \gamma y^{2}+\frac{3}{2}\left[\gamma\left(1-x^{2}-y^{2}\right)+2 x^{2}\right] \\
& -\sqrt{\frac{3}{2}}\left(\lambda_{1}+\lambda_{2}\right) x
\end{aligned}
$$

The critical points together with the stability analysis for constants $\lambda_{1}$ and $\lambda_{2}$ are shown in Table @ For completeness, the scalar field density parameter $\Omega_{\phi}$, the equation of state $\gamma_{\phi}$, the effective equation of state $\gamma_{e f f}$, the slope $p$, and the acceleration parameter $\bar{q}$ are shown in Table II. The eigenvalues of the stability matrix $M$, Eq. (A.1), are as follows:

- Point $\left(A_{ \pm}\right)$

$\left(x_{c}, y_{c}\right)=( \pm 1,0)$ is a kinetic dominated solution,

$$
\begin{aligned}
& m_{1}=3 \mp \sqrt{\frac{3}{2}}\left(\lambda_{1}+\lambda_{2}\right), \\
& m_{2}=3(2-\gamma) \mp \sqrt{6} \lambda_{1} .
\end{aligned}
$$

The fixed point $\left(A_{+}\right)$is stable for

$$
\lambda_{1}>\frac{6-\sqrt{6} \lambda_{2}}{\sqrt{6}}, \quad \text { and } \quad \lambda_{1}>\frac{3(2-\gamma)}{\sqrt{6}},
$$

and the fixed point $\left(A_{-}\right)$is stable for

$$
\lambda_{1}<-\frac{6+\sqrt{6} \lambda_{2}}{\sqrt{6}}, \quad \text { and } \quad \lambda_{1}<-\frac{3(2-\gamma)}{\sqrt{6}}
$$

- Point $(B)$

$\left(x_{c}, y_{c}\right)=\left(\left(\lambda_{1}+\lambda_{2}\right) / \sqrt{6},\left[1-\left(\lambda_{1}+\lambda_{2}\right)^{2} / 6\right]^{1 / 2}\right)$ is a scalar field dominated solution,

$$
\begin{aligned}
& m_{1}=-3+\frac{1}{2}\left(\lambda_{1}+\lambda_{2}\right)^{2}, \\
& m_{2}=-3 \gamma+\lambda_{2}\left(\lambda_{1}+\lambda_{2}\right) .
\end{aligned}
$$

The fixed point is a stable for

$$
\begin{aligned}
& -\sqrt{6}-\lambda_{2}<\lambda_{1}<\sqrt{6}-\lambda_{2}, \quad \text { and } \\
& \lambda_{1}>\frac{3 \gamma-\lambda_{2}^{2}}{\lambda_{2}} \text { for } \lambda_{2}<0, \quad \text { or } \\
& \lambda_{1}<\frac{3 \gamma-\lambda_{2}^{2}}{\lambda_{2}} \text { for } \lambda_{2}>0,
\end{aligned}
$$

- Point $(C)$

$\left(x_{c}, y_{c}\right)=\left((2 / 3)^{1 / 2} \lambda_{1} /(2-\gamma), 0\right)$ is a kinetic scaling solution,

$$
\begin{aligned}
& m_{1}=-\frac{3}{2}(2-\gamma)+\frac{\lambda_{1}^{2}}{2-\gamma}, \\
& m_{2}=\frac{3 \gamma}{2}-\frac{\lambda_{1} \lambda_{2}}{2-\gamma} .
\end{aligned}
$$


The fixed point is stable for

$$
\begin{aligned}
& \lambda_{1}^{2}>\frac{3}{2}(2-\gamma)^{2}, \quad \text { and } \\
& \lambda_{1}>\frac{3 \gamma(2-\gamma)}{2 \lambda_{2}} \quad \text { for } \quad \lambda_{2}<0, \quad \text { or } \\
& \lambda_{1}<\frac{3 \gamma(2-\gamma)}{2 \lambda_{2}} \quad \text { for } \quad \lambda_{2}>0 .
\end{aligned}
$$

- Point $(D)$

$\left(x_{c}, y_{c}\right)=\left((3 / 2)^{1 / 2} \gamma / \lambda_{2},\left[3 \gamma(2-\gamma) / 2 \lambda_{2}^{2}-\lambda_{1} / \lambda_{2}\right]^{1 / 2}\right)$ is a scalar field scaling solution,

$$
\begin{aligned}
& m_{1,2}=-\frac{3}{4}\left[2-\gamma\left(1+\frac{\lambda_{1}}{\lambda_{2}}\right)\right] \times \\
& {\left[1 \pm \sqrt{1-\frac{8\left(3 \gamma^{2}-6 \gamma+2 \lambda_{1} \lambda_{2}\right)\left(3 \gamma-\lambda_{1} \lambda_{2}-\lambda_{2}^{2}\right)}{3 \lambda_{2}^{2}\left[2-\gamma\left(1+\frac{\lambda_{1}}{\lambda_{2}}\right)\right]^{2}}}\right]}
\end{aligned}
$$

The fixed point is a stable for

$$
\begin{array}{ll}
\lambda_{1}<\frac{2-\gamma}{\gamma} \lambda_{2}, & \text { and } \\
\lambda_{1}<\frac{3 \gamma(2-\gamma)}{2 \lambda_{2}}, & \lambda_{1}>\frac{3 \gamma-\lambda_{2}^{2}}{\lambda_{2}},
\end{array}
$$

where $\lambda_{2}<0$,

$$
\begin{aligned}
& \lambda_{1}<\frac{2-\gamma}{\gamma} \lambda_{2}, \quad \text { and } \\
& \lambda_{1}>\frac{3 \gamma(2-\gamma)}{2 \lambda_{2}}, \quad \lambda_{1}<\frac{3 \gamma-\lambda_{2}^{2}}{\lambda_{2}}
\end{aligned}
$$

where $\lambda_{2}>0$.
[1] D. Colladay and V. A. Kostelecky, Phys. Rev. D 58, 116002 (1998) arXiv:hep-ph/9809521.

[2] S. M. Carroll, G. B. Field, and R. Jackiw, Phys. Rev. D 41, 1231 (1990).

[3] S. M. Carroll and E. A. Lim, Phys. Rev. D 70, 123525 (2004) arXiv:hep-th/0407149.

[4] B. M. Gripaios, JHEP 0410, $069 \quad$ (2004) arXiv:hep-th/0408127.

[5] T. Jacobson and D. Mattingly, Phys. Rev. D 64, 024028 (2001) arXiv:gr-qc/0007031.

[6] D. Mattingly, Living Rev. Rel. 8, 5 (2005) arXiv:gr-qc/0502097.

[7] V. A. Kostelecky and S. Samuel, Phys. Rev. D 39, 683 (1989).

[8] C. Eling and T. Jacobson, Phys. Rev. D 69, 064005 (2004) arXiv:gr-qc/0310044.

[9] E. A. Lim, Phys. Rev. D 71, 063504 (2005) arXiv:astro-ph/0407437.

[10] M. L. Graesser, A. Jenkins and M. B. Wise, Phys. Lett. B 613, 5 (2005) arXiv:hep-th/0501223.

[11] B. Z. Foster and T. Jacobson, Phys. Rev. D 73, 064015 (2006) arXiv:gr-qc/0509083.

[12] B. Z. Foster, Phys. Rev. D 73, 104012 (2006) arXiv:gr-qc/0602004.

[13] B. Li, D. F. Mota and J. D. Barrow, Phys. Rev. D 77, 024032 (2008).

[14] S. Kanno and J. Soda, Phys. Rev. D 74, 063505 (2006) arXiv:hep-th/0604192.

[15] E. J. Copeland, D. J. Mulryne, N. J. Nunes, and M. Shaeri1, arXiv:gr-qc/07081261].

[16] S. C. C. Ng, N. J. Nunes, F. Rosati, Phys. Rev. D 64,
083510 (2001).

[17] E. J. Copeland, A. R. Liddle and D. Wands, Phys. Rev. D 57, 4686 (1998) arXiv:gr-qc/9711068.

[18] S. Tsujikawa, Phys. Rev. D 73, 103504, (2006) arXiv:hep-th/0601178.

[19] L. Amendola, M. Quartin, S. Tsujikawa, and I. Waga, Phys. Rev. D 74, 023525, (2006) arXiv:astro-ph/0605488.

[20] I. Zlatev, L. Wang, and P. J. Steinhardt, Phys. Rev. Lett. 82, 896 (1999) arXiv:astro-ph/9807002; P. J. Steinhardt, L. Wang, and I. Zlatev, Phys. Rev. D 59, 123504 (1999) arXiv:astro-ph/9812313.

[21] B. Ratra and P. J. E. Peebles, Phys. Rev. D 37, 3406 (1988).

[22] L. R. W. Abramo and F. Finelli, Phys. Lett. B 575, 165 (2003) arXiv:astro-ph/0307208.

[23] J. M. Aguirregabiria and R. Lazkoz, Phys. Rev. D 69, 123502 (2004) arXiv:hep-th/0402190.

[24] E. J. Copeland, M. R. Garousi, M. Sami and S. Tsujikawa, Phys. Rev. D 71, 043003 (2005) arXiv:hep-th/0411192.

[25] Arianto, F. P. Zen, B. E. Gunara, Triyanta, and Supardi, JHEP 09, 048 (2007) [arXiv:hep-th/0709.3688].

[26] A. R. Liddle and R. J. Scherrer, Phys. Rev. D 59, 023509 (1998) arXiv:astro-ph/9809272.

[27] L. Wang, R. R. Caldwell, J. P. Ostriker and P. Steinhardt, Astrophys. J. 530, 17 (2000) arXiv:astro-ph/9901388.

[28] F. P. Zen and Arianto, in preparation. 